\title{
False Arrhythmia Alarms of Patient Monitoring Systems in Intensive Care Units
}

\author{
Erdem Yanar ${ }^{1}$, Yesim Serinagaoglu Dogrusoz $^{2}$ \\ ${ }^{1}$ ASELSAN, Ankara, Turkey \\ ${ }^{2}$ Middle East Technical University, Ankara, Turkey
}

\begin{abstract}
Generally in hospitals, intensive care units (ICUs) have high rates of false arrhythmia alarms independent of their brands and prices. These falsely issued alarms disrupt patients rest, drain hospital resources, and desensitize the hospital staff to potential emergency situations, which is named as false alarm fatigue. It has been estimated that $43 \%$ of life threatening electrocardiogram (ECG) alarms issued by bedside monitors are false, with some categories of alarm being as high as 90\%. In our study, we consider the alarms triggered by four life threatening conditions. These alarms are usually triggered by ECG and pulsatile waveforms recorded by monitoring equipment, which have standard alarm triggering criteria such as instantaneous thresholds on the predictor values. Most of the ICU false alarms are caused by single channel artifacts. In this study, we aim to fuse ECG features with information from other independent signals and get more robust alarm algorithms for ICUs. Pulsatile waveforms, which are highly correlated signals, can be used to corroborate the alarm category and to suppress significant number of false ECG alarms in ICUs. Photoplethysmogram (PPG), arterial blood pressure $(A B P)$ or both $P P G$ and $A B P$ can be used for this purpose. These waveformsare the least noisy pressure signal available in certain ICUs and rarely contain ECG-related artifacts. We implement four different algorithms that use information from ECG, PPG and $A B P$ waveforms, and compare the results.
\end{abstract}

\section{Introduction}

Electrocardiogram (ECG) analysis is now a routine monitoring tool for cardiovascular diseases, particularly in intensive care units (ICU). However, high false cardiac monitor alarm rates, mainly due to severe corruption by artifacts, noise and missing data, are extremely concerning. According to the publication of Lawless '[1]', the ICU false alarm (FA) rates might be so high as $86 \%$ for some arrhythmia types, with between $6 \%$ and $40 \%$ clin- ically insignificant, while only $6 \%$ alarms require immediate care. Poor performance of ICU monitoring devices results in care disruption, affecting both patients and medical staff by means of noise disturbances, desensitization to warning and longer response time. Furthermore, such disruptions have been shown to affect recovery of patients '[2]'.

Essentially, there are three kinds of technical approaches to help reduce false alarms '[3]': (1) improving signal extraction; (2) improving algorithms for alarm generation; (3) improving alarm validation. In recent years, several detection strategies have been applied to tackle this problem. For example, Aboukhalil et al. '[5]' and Deshmane '[6]' applied a multi-parameter analysis on ECG and pulsatile waveforms and signal quality assessment technology to improve algorithms for alarm generation. However, both methods met the problem that alarms had high true alarm (TA) suppression rate while low false alarm reduction rate because they only used morphological and timing information. The work described by Sayadi et al. '[6]' deployed a model-based filtering method to detecting alarms. Superior as the FA suppression rates are, this algorithm is computationally intensive. Qiao Li and Gari D. Clifford '[3]' extracted features from ECG, arterial blood pressure (ABP), and photoplethysmogram (PPG) and employed a machine learning approach. They achieved a ventricular tachycardia FA suppression of more than $30 \%$ with a true alarm suppression rate below $1 \%$.

Using the ECGII/V, PPG and ABP signals separately,validation was repeated 100 times randomly to examine the average performance of false alarm classifiers, and the results are shown in Table III. This algorithm showed good performance on AS false alarm suppression with high scores and validation accuracy. The AS alarms are classified well with $83 \%$ sensitivity, $78 \%$ specificity. The BC alarms are classified well with $93 \%$ sensitivity, $69 \%$ specificity. Overall, in comparison with the top candidate entries of the 2015 PhysioNet/Computing in Cardiology Challenge, this algorithm shows promising results, as is demonstrated by Table 2 . 
Table 1. Sensitivity and Specificity Values.

\begin{tabular}{lcr}
\hline \hline EKG1 & Sensitivity & Specificity \\
\hline \hline Asistoli & $\% 81$ & $\% 90$ \\
\hline Bradikardi & $\% 97$ & $\% 76$ \\
\hline \hline EKG2 & Sensitivity & Specificity \\
\hline Asistoli & $\% 68$ & $\% 85$ \\
\hline Bradikardi & $\% 93$ & $\% 78$ \\
\hline \hline EKG1+ABP+PPG & Sensitivity & Specificity \\
\hline \hline Asistoli & $\% 82$ & $\% 79$ \\
\hline Bradikardi & $\% 97$ & $\% 69$ \\
\hline \hline EKG2+ABP+PPG & Sensitivity & Specificity \\
\hline \hline Asistoli & $\% 83$ & $\% 78$ \\
\hline Bradikardi & $\% 93$ & $\% 69$ \\
\hline \hline
\end{tabular}

\section{MATERIALS AND METHODS}

\subsection{Signals Preprocessing Stage}

The preprocessing step starts by retrieving the input signals. The signals from two ECG leads are used in this work as well as the ABP and PPG signals. The algorithm rst searches for ECG leads II and V and uses any available leads if these two are not available. All the unavailable signals are set to zeros. Only 16 seconds prior to the alarm are used in this work and the rest of the signals are discarded. All the signals are re-sampled to $125 \mathrm{~Hz}$. The retrieved signals are then ltered using zero-phased band-pass finite impulse filter designed with Hamming window approach between $5 \mathrm{~Hz}$ and $40 \mathrm{~Hz}$ for the ECG signals and between 0.5 and $10 \mathrm{~Hz}$ for the ABP and PPG signals. The output was differentiated by using a 2 point first-order digital differentiator and then applied a sample by sample nonlinear squaring operation. These two operation is equivalent to applying a high pass filter then applying a low pass one. In this way $\mathrm{P}$ and $\mathrm{T}$ waves are attenuated while amplfying higher frequencies like $\mathrm{R}$ wave. In the last stage of preprocessing the signal was analysed for non physiological parts, such as flat baseline signals, NAN signal values and extremely noisy parts because of poor electrode contacts. If any of these condtions identified ECG signal marked as not reliable and does not enter any ECG detection algorithm.

\subsection{Heart Beat Detection}

Accurate detection of heart beat may potentially contribute to suppressing false arrhythmia alarms. Therefore, a robust heart beat detection algorithm was employed to derive characteristic point positions from multiple physiological signals.
Derivate-based algorithms: these algorithms based in filters and derivative as in [4]. They often use a high-pass filter for pre-processing stage and in the final stage derivative is used to determine the maximum slope, which corresponds to QRS complex.

Algorithms based on digital filters: these algorithms use more sophisticated filters [4]. Two different filters process the ECG in the pre-processing stage, low-pass and high pass ones, with different cut-off frequencies, forming the band-passfiltered signal. In the final stage also thresholds are compared adaptively.

Wavelets: in wavelet based approaches discompose the signal into different scale components to analyze the signal in different frequency bands like pre-processing in a different way. Then fixed thresholds are applied to obtain the characteristic points in final stage.

Neural Networks: neural networks are used to predict current signal values from the past ones. Therefore they apply suitable filters to attenuate the noise.

Hidden Markov Models: HMMs model the data sequence according to an underlying Markov chain. The algorithm infers the underlying state from the observed signal.

Genetic algorithms: in this algorithm they intend to get optimal polynomial filters, for preprocessing stage. Moreover, it get parameters for decision stage.

Phasor Transform: this algorithm transforms each sample of the signal into a complex value, which preserves the signal information. It enhances ECG waves and then the detection is easier by applying thresholds.

At end of our trials we chose Hilbert transform method as our QRS detection algorithm. One of the properties of the Hilbert transform is that it is an odd function. That is to say that it will cross zero on the $\mathrm{x}$-axis every time that there is an inflexion point in the original waveform(Fig. 1). Similarly a crossing of the zero between consecutive positive and negative inflexion points in the original waveform will be represented as a peak in its Hilbert transformed conjugate. This interesting property can be used to develop an elegant and much easier way to and the peak of the QRS complex in the ECG waveform corresponding to a zero crossing in its first differential waveform $\mathrm{d} / \mathrm{dt}(\mathrm{ECG})$. The block diagram of the proposed approach is shown in Figure 2. This method fused $\mathrm{R}$ peak positions detected on the ECG using an adaptive thresholding and onset positions 
detected on the pulsatile waveforms (ABP and/or PPG) using length transform.

\subsection{Feature Extraction}

ABP Signal:Three PhysioNet open-source algorithms were used to process the arterial blood pressure (ABP). The wabp algorithm was applied to ABP signal to detect the onset points of the pulses in the signal [1]. This algorithm is based on the length transform [1]. The abpfeature algorithm was then applied to extract features from ABP signal such as systolic and diastolic pressure, systolic area, and mean pressure at each detected pulse. The jSQI algorithm was implemented to investigate the signal quality of each beat of ABP signal. It is based on removing the features and onset points that are not physiologically meaningful. Finally, the RR-intervals were calculated as the difference of the onset points of the pulses in the signal.

PPG Signal:Three PhysioNet open-source algorithms were used to process the photoplethysmogram (PPG) signal. The quantile algorithm was applied to partition the signal into three quantiles, $(0.05,0.5,0.95)$. The wabp was used for onset point detection and was applied to the subtraction of third quantile and first quantile. Then, the RR-intervals were calculated from the onset points. The ppgSQI algorithm was used to estimate the signal quality index based on beat template correlation.

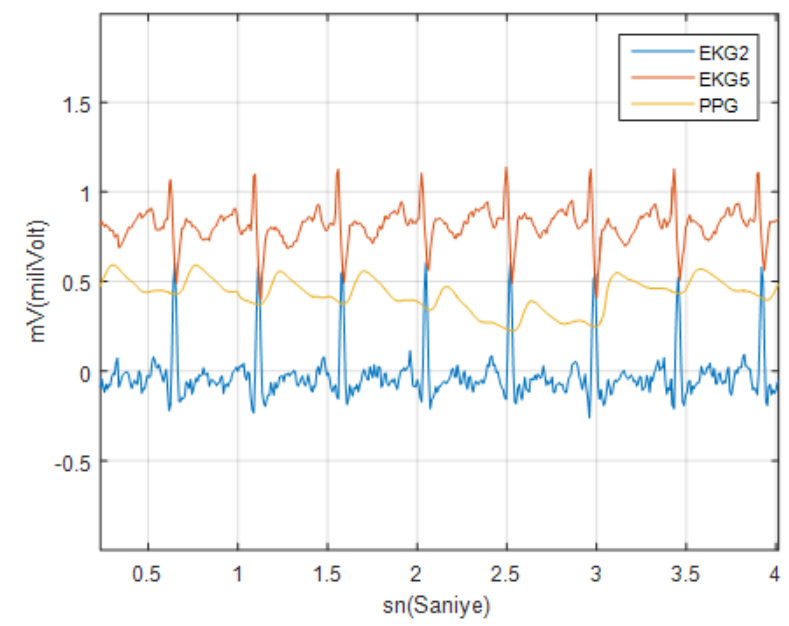

Figure 1. ECG Signal Preprocessing

\subsection{Feature Selection}

Asystole:Asystole was defined as no heart beat for at least four seconds. Therefore, the minimum Asystole threshold was set to four seconds with a tolerance of 0.5 .

Bradycardia: Bradycardia was defined by the heart rates of less than $40 \mathrm{bpm}$ for five consecutive beats. Therefore, minimum heart rate for each five beats through the segment was measured and if it passed this threshold, the alarm as set to false.

\subsection{Alarm Decision}

In this context, all signals were entered to preprocessing stage, heart beat detection, feature extraction and feature selection stages.the algorithm checked if the ECG signal was available and if the signal quality was adequate, the maximum RR-interval was compared to the asystole threshold. If it was greater, then the alarm was set to False. If the ECG signal was not available or the signal quality was not in the proper range. Then, the algorithm will go through ABP signal and then PPG signal to check the maximum RR-interval compared to the threshold. Moreover, our fuzzy logic algorithm parameters given as SQI_th=0.85, AS_th=4, BC_th=40 and demonstrated on Figure 2.



Figure 2. Alarm Decision Algorithm 


\section{RESULTS}

Using the 13 most selected features, fivefold cross validation was repeated 50 times randomly to examine the average performance of false alarm classifiers, and the results are shown in Table III. This algorithm showed good performance on AS and BC false alarm suppression, while classifiers achieved very high scores, both of the corresponding cross validation are moderate. Overall, in comparison with the top candidate entries of the 2015 PhysioNet/Computing in Cardiology Challenge, this algorithm shows promising results, as is demonstrated by Table 2 .

Table 2. Results

\begin{tabular}{lcr}
\hline \hline Algorithm(Asystole) & TPR(\%) & TNR(\%) \\
\hline Plesinger et al. & 92 & 88 \\
\hline Fallet et al. & 94 & 77 \\
\hline Ansari et al. & 94 & 82 \\
\hline Sadr et al. & 78 & 93 \\
\hline This Work & $\mathbf{8 3}$ & 93 \\
\hline
\end{tabular}

\section{CONCLUSION}

Data fusion and signal quality index provide an accurate detection of heart beat, which is proved to help in a decision-making process. Though the selected features are defined previously, their combination describes properties from various aspects and may reveal more difference between true and false alarms. Among the features, the HRV analysis parameters lift the performance of alarm classifiers to a great extent. Besides, the average time for extracting features. From a recording is 1.63 second, thus, the alarm classification will be finished within 2 seconds when an alarm is triggered. However; if we use another test set we should see a reduction in our sensitivity due to being tuned to the training set rather than being generalized. This is a typical problem with developing any analysis algorithm or logic. When the sensitivity was improved by including arterial blood pressure and pulse data, this improvement was offset by a reduction in specificity. In other words, the improved detection of clinical conditions was offset by an increased number of false alarms. The percentage point decrease in specificity (increasing false alarm rate) was greater than the percentage point increase in detection of true clinical alarm conditions. Similar results are expected to present in other arrhythmias, such as atrial fibrillation, and further work will be concentrated on clinical experiments and applications.

\section{References}

[1] Clifford GD, Silva I, Moody B, Li Q, Kella D, Shahin A, Kooistra T, Perry D, Mark RG. The PhysioNet/Computing in Cardiology Challenge 2015: Reducing False Arrhythmia Alarms in the ICU. In Computing in Cardiology, 2015. September 2015;

[2] Clifford GD, Silva I, Moody B, Li Q, Kella D, Shahin A, Kooistra T, Perry D, Mark RG. The PhysioNet/Computing in Cardiology Challenge 2015: Reducing False Arrhythmia Alarms in the ICU, 2015. September 2015.

[3] Clifford GD. Signal Processing Methods for Heart Rate Variability. Ph.D. thesis, University of Oxford, 2002

[4] Nygards ME, Sornmo L. Delineation of the QRS Complex using the Envelope of the ECG. Med and Biol Engineering and Computing 1983;21(5):538547

[5] AAboukhalil, L Nielsen, M Saeed, R G Mark, and G D Clifford. Reducing false alarm rates for critical arrhythmias using the arterial blood pressure waveform. Journal of Biomedical Informatics, 41(3):442451, 2008.

[6] W Zong, G Moody, R Mark, Reduction of false blood pressure alarms bybuse of electrocardiogram blood pressure relationships. Comput. Cardiol.26, 305308 (1999)

[7] James Sun Feb 092005 with some changes from Gari Clifford based upon wabp.c by Wei Zong (www.physionet.org)

Address for correspondence:

Erdem Yanar

Mehmet Akif Ersoy Mahallesi 296. Cadde

No:16, 06370 Yenimahalle-Ankara TURKEY

eyanar.bme@gmail.com

Assoc. Dr. Yesim Serinagaoglu Dogrusoz

Middle East Technical University

Electric and Electronics Engineering Department

Cankaya-Ankara TURKEY

yserin@metu.edu.tr 